J Child Adolesc Psychopharmacol Vol. 19(4):453-460 (2009)

ISSN: (print 1044-5463)(online 1557-8992)

doi:10.1089/cap.2008.0157

This is a peer reviewed pre-print version of the following article: Multinutrient Supplement as Treatment: Literature Review and Case Report of a 12-Year-Old Boy with Bipolar Disorder, which has been published in final form at: http://www.liebertpub.com

http://www.liebertpub.com/cap

http://www.liebertonline.com/doi/pdfplus/10.1089/cap.2008.0157

(C) 2009 Mary Ann Liebert, Inc. Publishers

\title{
Multinutrient Supplement as Treatment: Literature Review and Case Report of a 12-Year-Old Boy with Bipolar Disorder
}

\author{
Elisabeth A. Frazier, B.S., Mary A. Fristad, Ph.D., ABPP, and L. Eugene Arnold, \\ M.D., M.Ed.
}

\begin{abstract}
Early-onset bipolar disorder has significant morbidity and mortality. Development of safe, effective treatments to which patients will adhere is critical. Pharmacologic interventions for childhood bipolar spectrum disorders are limited and are associated with significant risk for adverse events. Diet and nutrition research suggests vitamins, minerals, and other nutrients are important underpinnings of general physical and mental health; furthermore, they may even be useful in treating mood dysregulation by providing a more favorable risk-benefit ratio than contemporary psychotropic agents. This article reviews the literature on multinutrient supplementation and mental health, and examines a case study of a 12-year-old boy with bipolar disorder and co-morbid diagnoses treated for 6 years with conventional medication and finally a multinutrient supplement. The multinutrient supplement in this case study is EMPowerplus (EMP+), a 36ingredient supplement containing 16 minerals, 14 vitamins, 3 amino acids, and 3 antioxidants. It was used to treat a 12-year-old boy initially diagnosed with bipolar disorder not otherwise specified (BP-NOS) at age 6 , and whose diagnosis evolved by age 10 to bipolar I (BP-I), mixed, with psychotic features. He also met criteria for generalized anxiety disorder by age 8 and obsessive-compulsive disorder by age 10. After 6 years of conventional treatment (ages 6-12), he received 14 months of EMP+. Symptom manifestation over 7 years is described in conjunction with treatment history.EMP+ resulted in outcome superior to conventional treatment. This report adds to accumulating preliminary evidence that further basic science and clinical studies of multinutrient supplements are warranted.
\end{abstract}

\section{Introduction}

Childhood-onset bipolar spectrum disorders (BPSD; bipolar I [BP-I], bipolar II [BP-II], Bipolar not otherwise specified [BP-NOS], and cyclothymia) represent a significant public health concern (McClellan et al. 2006; Brown et al. 2008). Although recent clinical trials have found efficacy in using atypical antipsychotics for BP-I, treatment literature is lacking for children with BP-II, BP-NOS, and cyclothymia, despite the functional impairment inherent to these diagnoses (Kowatch et al. 2009). Medications recommended in current treatment guidelines (Kowatch et al. 2005; McClellan et al. 2006) appear beneficial but carry significant risk for adverse events.

In a recent retrospective study of medical and pharmacy claims from a cohort of 4140 youths prescribed one of five types of atypical or two conventional antipsychotics compared to a random sample of 4500 youth not treated with psychotropics, the treated cohort had higher rates of a variety of metabolic and cardiovascular side effects (McIntyre and Jerrell 2008). These adverse events included higher risk of obesity, type 2 diabetes mellitus, cardiovascular conditions, dyslipidemia, and orthostatic hypotension. Researchers found metabolic and cardiovascular risks increased for youths taking multiple antipsychotics (McIntyre and Jerrell 2008). 
In another study monitoring side effects of atypical neuroleptics, including clozapine, olanzapine, and risperidone, all three drugs caused drowsiness and hypoactivity. A total of 30$60 \%$ of children and adolescents taking clozapine experienced constipation, increased salivation, orthostatic hypotension, and nasal congestion. These side effects were seen in patients taking olanzapine and risperidone less often, but 5-15\% of participants taking olanzapine or risperidone suffered from rigidity, tremor, and dystonia. Participants in all three atypical neuroleptic conditions gained weight during the study, but those in the olanzapine group gained significantly more weight than those in the other two treatment groups (4.6 $\pm 1.9 \mathrm{~kg}$; Fleischhaker et al. 2006).

Recent clinical trials of depression and bipolar disorders in youth show approximately 20-25\% of participants dropped out of psychotropic medication treatment (Biederman et al. 2007; DelBello et al. 2007). DelBello and colleagues (2007) conducted a single-blind, 12-week study of quetiapine in adolescents aged 12-18 in which researchers observed a 25\% drop out rate of adolescents whose diagnoses included dysthymia, BP-II, BP-NOS, major depressive disorder, or cyclothymia. Biederman and colleagues (2007) conducted an 8-week, open-label trial of aripiprazole in children aged 6-17 with BP in which they observed a 21\% drop out rate. Additionally, a recent study of an anticonvulsant mood stabilizer in children failed to show any superiority to placebo (Wagner et al. 2006).

\section{Dietary supplementation and mental health}

Previous research on diet and nutrition suggests multinutrient supplements may have a beneficial effect on mood with limited side effects, which might provide a primary treatment with a more favorable risk-benefit ratio for some youth suffering from BPSD than currently available pharmacologic interventions (Kaplan et al. 2001; Popper 2001; Kaplan et al. 2002; Kaplan et al. 2004; Kaplan et al. 2007). Increasing evidence suggests that nutrition affects the structure and functioning of the brain due to the high percentage of human metabolic activity accounted for by this organ. In adulthood, the brain accounts for $20 \%$ of the human basal metabolic rate; as a neonate, this number is as high as 44\% (Benton 2008). Nutritional interventions, particularly multi-ingredient multinutrient supplements, have several possible mechanisms of action to explain a hypothesized association with clinical improvement in mood. These are summarized below. Preliminary studies have been conducted with the multinutrient supplement EMPowerPlus (EMP+) (Truehope Nutritional Support Ltd., Raymond, Alberta, Canada). This supplement consists of 16 minerals, 14 vitamins, 3 amino acids, and 3 antioxidants (a full list of ingredients can be found on the manufacturer's website at http://www.truehope.com). Relevant literature is discussed below.

Individual vitamins and minerals.

The neuropsychiatric effects of certain frank nutritional deficiencies are well known: e.g., thiamine/B1 (Wernicke encephalopathy), niacin/B3 (pellagra), cyanocobalamin/B12 (psychosis of pernicious anemia), and iodine (myxedema madness). Milder effects of more subtle deficiencies are increasingly recognized, possibly related either to genetic variations in which some patients may be more vulnerable or to historical changes in diet composition. Nutritional supplements have been related to a wide range of human health factors from neuronal development to depression (Hibbeln 1998; Noaghiul and Hibbeln 2003). Specific nutrients linked to mental health include iron, copper, zinc, vitamins $\mathrm{B}_{1}, \mathrm{~B}_{6}, \mathrm{~B}_{12}, \mathrm{D}$, E, and folate (Kaplan et al. 2007).

\section{Multinutrient combinations.}


Recently, interest has turned to combinations of nutritional deficiencies, because there is plausible reasoning to support the concept that if one nutrient is deficient, a grouping of nutrients are deficient, and the level of one nutrient can affect the adequacy of others (Benton 2008; Kaplan et al. 2007). Kaplan and colleagues (2007) review studies of vitamins and minerals and their relationship with mood symptoms, then present four conceptual frameworks that are compatible, can coexist, and may aid understanding of how nutrients may improve mood (Kaplan et al. 2007). First, they suggest mood dysregulation may result from innate metabolism malfunctions, ultimately affecting brain functioning; such innate mechanisms could involve inefficient use of nutrients. Second, they state mood instability may result from deficiencies in methylation of molecules responsible for completing DNA transcription, switching on genes, regulating protein generation, activating enzymes, and synthesizing neurotransmitters. Third, nutrition deficiencies may alter gene expression, leading to mood instability. Fourth, unstable mood may result from long-latency effects of nutrient deficiencies that alter brain development directly or by way of dysfunctional nutrient absorption. Although these four frameworks require considerable empirical evaluation, they provide possible mechanisms through which nutrient supplementation may affect mood symptoms (Kaplan et al. 2007).

Gesch et al. (2002) examined the effects of vitamins, minerals, and essential fatty acids on antisocial behavior in a randomized, double-blind, placebo-controlled study of 231 adult prisoners. Participants spent, on average, 142 days on the recommended daily dose of two supplements (one capsule of Forceval, a vitamin-mineral supplement that contains 25 vitamins and minerals, and four capsules of Efamol Marine, an essential fatty acid supplement containing omega-6 and omega-3 essential fatty acids) or placebo (identically appearing oil-based gelatin capsules). The Efamol Marine dose contains $1260 \mathrm{mg}$ of linoleic acid, $160 \mathrm{mg}$ of gamma linolenic acid, 80 of eicosapentaenoic acid, and $44 \mathrm{mg}$ of docosahexaenoic acid (Gesch et al. 2002).

Participants' antisocial behavior was measured throughout the study period using disciplinary reports. Results revealed a decrease in overall antisocial behavior for participants taking nutritional supplements compared to placebo. Neither group reported notable side effects. Overall infringements resulting in disciplinary reports decreased by $35.1 \%$ in the active condition for participants who took the supplement for at least 2 weeks compared to placebo participants, whose disciplinary reports decreased by 6.7\% $(p<0.001)$. Intent-to-treat analyses showed active condition participants experienced a $26 \%$ decrease in overall infringements resulting in disciplinary reports compared to placebo participants $(p<0.03)$. This research suggests a combination of vitamin-mineral supplements and essential fatty acids may decrease antisocial behavior; the researchers speculate that physiological changes caused by dietary intervention affect mental health and warrant further clinical investigation (Gesch et al. 2002).

Schoenthaler and Bier (2000) examined the impact of low-dose vitamin-mineral tablets on rates of violent, antisocial behavior in 468 school children aged 6-12. Using a stratified randomized double-blind, placebo-controlled design, half the sample received daily vitaminmineral supplementation at $50 \%$ of the U.S. recommended daily allowance (RDA) for 4 months. The other half received placebo. Eighty children were disciplined at least once during the September 1 to May 1 study interval. The 40 who received supplementation had a $47 \%$ lower mean rating of antisocial behavior than the 40 on placebo (1 vs. 1.875 disciplinary actions). Children on supplementation had lower antisocial behavior ratings for every type of recorded infraction: Threats/fights, vandalism, disrespect, disorderly conduct, assault/battery, defiance, obscenities, refusal to work/serve, endangering others, and nonspecified offenses. 


\section{EMP+ Multinutrient supplement.}

As indicated above, there is clear scientific rationale for studying multinutrient formulations. Early clinical findings suggest multinutrient supplements may have promising therapeutic effects in patients with BP (Kaplan et al. 2001; Kaplan et al. 2002; Simmons 2003). EMP+ is a commercially available product with the most extensive foundation of empirical support to date. Background research on this product, summarized below, suggests it is worthy of more rigorous scientific evaluation.

\section{Animal study.}

Halliwell and Kolb (2003) studied newborn rats who received frontal or posterior parietal lesions on day 3 , then subsequently were fed either normal rat chow or rat chow enhanced with a rodent-appropriate dose of EMP+. At day 60, the supplemented animals exhibited reversal of behavioral deficits (e.g., performance on spatial learning tasks) and had significant regrowth of cortical tissue compared to unsupplemented rats. Behaviorally, the animals were significantly calmer than unsupplemented rats.

\section{Adult studies.}

Initial studies were conducted with adult patients resistant to conventional treatments. Open-label trials were first reported on adults diagnosed with BP (Kaplan et al. 2001). Kaplan and colleagues studied 11 patients aged 19-46 years for 6-21 months. The effects of EMP+ on symptoms of bipolar disorder were assessed using the Hamilton Depression Rating Scale (HAMD) (Hamilton 1960), the Young Mania Rating Scale (YMRS) (Young et al. 1978), and the Brief Psychiatric Rating Scale (BPRS) (Overall and Gorham 1962). Participants could continue using concurrent psychiatric medications under the supervision of their psychiatrist. Results indicated a 55-66\% reduction in symptoms reported on the HAM-D, YMRS, and BPRS as well as a 50\% decrease in the need for psychotropic medications. Participants reported one mild side effect, infrequent/transitory nausea, which occurred most commonly when participants took their supplement without food (Kaplan et al. 2001). The researchers recommended further empirical investigation of EMP+ by other researchers.

Simmons (2003) described his use of EMP+ in private clinical practice. He reported that 12 out of 19 treatment-resistant adult patients diagnosed with BP-I $(n=14)$ and BP-II $(n=5)$ who began what is now an outdated version of EMP+ displayed marked improvement; 3 appeared moderately improved and 1 person showed mild improvement after a mean of 5.3 weeks on the supplement. Thirteen participants completely stopped taking their original psychiatric medications after an average of 5.2 weeks on EMP+ (range $=3-10$ weeks) and remained stable. Side effects included mild gastrointestinal problems, but a majority of patients (11 of 19, 58\%) continued using this supplement instead of their previously prescribed psychopharmacological medications (Simmons 2003). While placebo-controlled trials are currently being conducted by Kaplan and colleagues in adults, placebo-controlled trials have not yet commenced with children (Kaplan et al. 2001). However, several case series and open trial studies have been conducted, reviewed below.

Child studies.

Several studies examine the efficacy of EMP+ in children. In a naturalistic ABACB trial, Popper (2001) followed a 10-year-old boy diagnosed with BP who experienced severe temper tantrums multiple hours a day for 4 months in his clinical practice. After 2 days of taking EMP+ 
at full dose, the boy's behavior improved significantly. Within 5 days, all tantrums and irritability ceased. After 14 days, EMP+ was discontinued. Within 2 days of discontinuation, tantrums began again. The boy was then put on a different supplement, which, according to parent and teacher reports, provided $60 \%$ of the benefit noted on EMP+. EMP+ was resumed a second time, resulting in resolution of the tantrums and irritability. In a follow-up study, Popper followed 22 more patients with BP in his clinical practice (2001). Participants included 10 adults, 9 adolescents, and 3 preadolescents. Although mild side effects were common (e.g., headache), a majority of participants (19/22, 86\%) responded positively. Furthermore, 11 of the 15 patients (73\%) previously on psychotropics remained stable without resumption of these medications at 6-month and 9-month follow-up assessments (Popper 2001).

Kaplan and associates also conducted an open-label ABAB trial with 2 boys aged 8 and 12 (Kaplan et al. 2002). These participants displayed irritability, mood lability, and explosive rage at baseline. The 8-year-old boy had diagnoses of atypical obsessive-compulsive disorder (OCD) and attention-deficit/hyperactivity disorder (ADHD). The 12-year-old boy was diagnosed with pervasive developmental disorder (PDD). The 8-year-old displayed consistent explosive rage, irritability, and obsessions with guns (but no compulsions) during baseline and each withdrawal phase. When on EMP+ these behaviors were almost completely eliminated. His obsessive thoughts ceased, the frequency and duration of his temper outbursts decreased significantly, and his mood fluctuations minimized. After more than 2 years of treatment, the boy remained well and free of side effects while taking $25 \%$ of his initial dose of EMP+. The second boy, 12 years old, displayed consistent irritability, negative attitude, temper outbursts, and extremely disruptive behavior in school at baseline. These behaviors subsided markedly while on EMP+. His mood impairment and temper levels returned to baseline status during treatment withdrawal. He demonstrated marked improvement in mood stabilization and behavior when treatment was reintroduced. Stimulant medication was still required in addition to EMP+ to control ADHD symptoms. After almost 3 years of treatment, this boy also maintained wellness without adverse side effects on $25 \%$ of his original dose (Kaplan et al. 2002).

Kaplan and colleagues also completed a case series to further test the impact of EMP+ in 11 children ages 8-15 (Kaplan et al. 2004). All had mood and/or behavioral problems. Diagnoses at intake included: BP ( $n=3$; 1 co-morbid with anxiety, 1 with behavior disorders); Asperger plus an anxiety disorder ( $n=2)$; ADHD+ co-morbid anxiety and/or behavior disorders $(n=5)$; and Praeder-Willi syndrome, ODD+ anxiety $(n=1)$. Nine of the $11(82 \%)$ completed the openlabel trial. Intent-to-treat analyses indicated significantly decreased scores on the Youth Outcome Questionnaire (YOQ) $(p<0.001)$ and YMRS $(p<0.01)$ from baseline to final visit. For the 9 completers, improvement was significant on 7/8 (88\%) Child Behavior Checklist scales, YOQ and YMRS $(p<0.05$ to $p<0.001)$. The authors concluded that results from this study support the need for formal clinical trials of nutritional interventions in children with mood and behavioral dysregulation (Kaplan et al. 2004).

It is important to note all above publications used a now-outdated version of EMP+, one that often caused gastrointestinal side effects. In the new version, the ingredients are processed differently; in particular, the minerals are pulverized so the individual particle size has been significantly reduced to as low as 14-15 microns. Consequently, the ingredients now look like powder and pack into fewer capsules. There have also been some small reductions in quantities of several vitamins (e.g., vitamin A). This updated version is the one used in the intervention described below.

\section{Case Presentation}


This case presentation follows the treatment of a 12-yearold boy ("John”) diagnosed with BP-NOS, which later developed into BP-I with psychotic features, generalized anxiety disorder (GAD), and OCD. Enuresis also emerged while taking lithium. By age 6, John experienced severe mood cycling, sadness, irritability, self-harming behaviors, sleep disturbance, and severe tantrums. He also experienced elevated mood, poor peer relations, low frustration tolerance, flight of ideas, aggressive behavior, hyperactivity, and impulsive negative behaviors. By age 8 , John developed impairing anxiety and worsening mood symptom intensity and cycling, increased destructive behavior, transient suicidal ideation, and increased global impairment. These symptoms continued as he grew older, and between ages 10 and 11, John began experiencing auditory hallucinations, developed obsessions and compulsions, and displayed increasingly disrespectful and aggressive behaviors. He reported hearing intrusive, commanding voices when he became anxious, telling him to act on his obsessions and do things he did not want to do, and threatening him. At one point, John reported hearing voices almost 100 times in one week telling him, "If you don’t do this you'll surely die.” and "Don’t listen to them [referring to parents and other adults]," When he was 10 years old, John specifically noted the voices told him he would die on the day John Glenn dies. John's symptoms became so impairing he was removed from his private school and transferred to home schooling.

John's treatment team frequently altered his medications from the time he was 6 years old until he was 12 years old (2001-2007) in conjunction with individual/family psychoeducational psychotherapy (Fristad et al., in press ) provided by the second author (see Table 1). Medication changes were due to intolerable side effects and/or inadequate treatment response. During this time, he took various combinations of prescription and over-the-counter agents, including lithium citrate, risperidone, lithium carbonate, clonidine, flax seed, desmopressin, omega-3 fatty acids, magnesium, trazodone, gabapentin, valproic acid, propranolol, quetiapine, aripiprazole, lorazepam, and lamotrigine. No medication, alone or in combination, maintained a desirable mood balance or consistent improvement in global functioning over an extended period of time.

When John was 12 years old, his mother approached his therapist (the second author) asking about EMP+. Coincidentally, the authors were intending to begin an open-label trial of EMP+ at their treatment center; however, there was a considerable delay in commencing the study due to impediments in receiving all research approvals needed. Thus, rather than wait, the family chose to work independently with the Truehope staff in starting EMP+ and received directions to taper psychotropic medication from his child and adolescent psychiatrist. The family continued to report John's progress to his therapist over the subsequent 14 months.

John's transition from psychotropic medication to EMP+ is chronicled in Table 2. John took 5 capsules per day, then 10 per day, and then the recommended 15 capsules per day. After 7 days on EMP+, he began to reduce his lithium carbonate and lamotrigine as recommended by the Truehope consultant and his psychiatrist. After 19 days on EMP+, John was completely off all psychotropic medications.

Throughout this tapering-and-titration process, John experienced transient episodes of irritability, headache, dizziness, and fatigue. However, his global functioning notably increased. As treatment continued, John began interacting more appropriately with peers, remained calm and playful throughout most of the day, slept throughout the night, remained focused and efficient while completing schoolwork, and experienced decreased compulsions. Hallucinations ceased. John continued to experience brief periods in the afternoon when he would become frustrated, irritable, or defiant with his parents. However, his parents reported these behaviors were consistently followed by a sincere apology, which they viewed as an improvement 
compared to previous behavior. The night terrors and previously mentioned side effects ceased, his bowel movements normalized, his skin appeared smooth without dry patches, and his symptoms remained stable. His anxiety decreased, functioning was no longer impaired at home, with schoolwork, or with peers, and his impulsivity and fidgeting decreased substantially. All diagnoses fully remitted.

\section{Extended follow-up information}

After 4 months of EMP+, John's mother increased his dose from 15 capsules a day to 18 capsules to manage emerging irritability, possible mood cycling, and “odd" behavior. She reported this increase relieved his symptoms. One month later, the dose was returned to 15 capsules of EMP+ daily. John has maintained health over the subsequent 9 months. He enrolled in regular public school for the first time, where he plays successfully on the school soccer and baseball teams and maintains friendships. His parents subjectively report that improvements in his functioning while taking EMP+ are greater than those he made in the past on other medications. His only additional interventions are drinking a half cup of whey protein mixed in milk in the morning during sports seasons while very active and using a lightbox during the winter. According to his mother, EMP+ helps keep him "clear, slowed down, peaceful, settled and happy."

\section{Discussion}

Pharmacological intervention is considered to be the firstline treatment for children with $\mathrm{BP}$, and recent reviews of the research suggest mood stabilizers or atypical antipsychotics should be the first psychotropics considered for children with BP-I (Kowatch, et al. 2009).

Unfortunately, research on other psychotropic medications and varying presentations of pediatric bipolar disorder (BP-II, BP-NOS, cyclothymia) is limited, and those agents found efficacious are associated with risk for adverse events (Kowatch et al. 2005). It may take an average of 9 months to 2 years until an efficacious drug combination is found to stabilize mood in youths, and relapse rates are high (Kowatch et al. 2009). The Food and Drug Administration (FDA) recently approved the use of risperidone and aripiprizole in children with BP, although many children experience significant metabolic side effects on therapeutic doses (Slatko 2007). Lithium is another FDA-approved psychotropic medication for youths as young as 12 years, and it also is associated with numerous adverse side effects. Developing safe and effective treatments for early-onset BP to which patients will adhere is critical. 


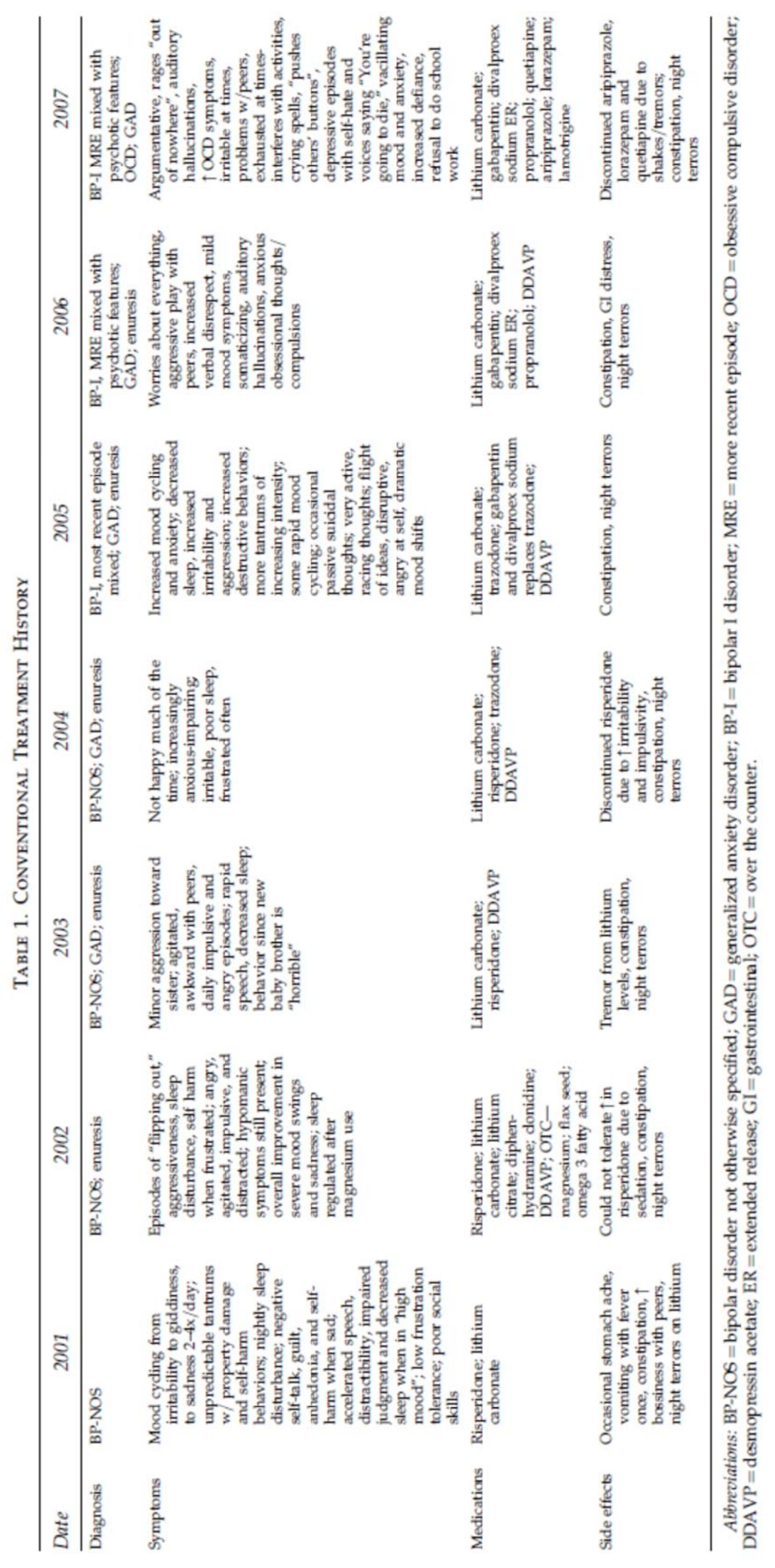




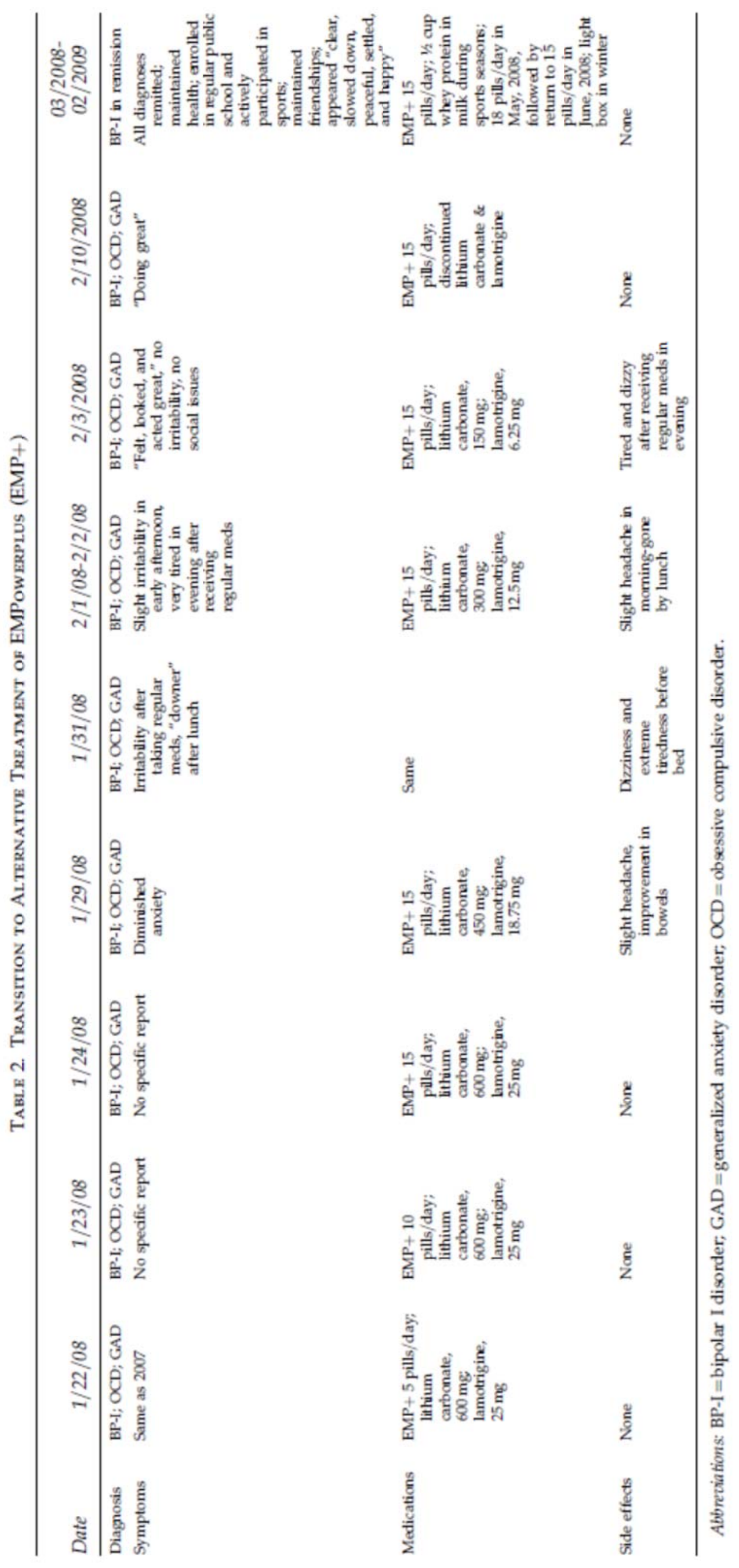

